\title{
QUALIDADE MICROBIOLÓGICA DE REQUEIJÃO E DOCE DE LEITE PASTOSO PRODUZIDOS EM TOCANTINS - MG
}

\author{
Cleuber Raimundo da Silva \\ Mestre em Ciência dos Alimentos/UFLA/MG \\ Professor do Setor de Produção Alimentícia do Instituto Federal de Educação, Ciência e Tecnologia de Santa \\ Catarina - Campus Canoinhas (IF SC Campus Canoinhas) \\ cleubertla@hotmail.com
}

\section{João Batista Barbosa}

Doutorando em Produção Vegetal/Laboratório de Tecnologia de Alimentos/UENF/RJ

joaotla@yahoo.com.br

\section{Fernanda Cristina Firmino}

Mestranda em Ciência e Tecnologia de Alimentos/Departamento de Tecnologia de Alimentos/UFV/MG fernandacristinafirmino@yahoo.com.br

\section{Leandro Lamas Caldoncelli}

Tecnólogo em Laticínios/IF Sudeste MG

Técnico Administrativo do Instituto Federal de Educação, Ciência e Tecnologia Sudeste de Minas Gerais (IF Sudeste MG, Campus Rio Pomba)

leandro.lamas@ifsudestemg.edu.br

\section{RESUMO}

O requeijão é um produto tipicamente brasileiro, fabricado em todo o território nacional, com algumas variações de tecnologia e características. Outro produto de grande importância é o doce de leite pastoso, o qual a produção no Brasil é feita por muitas empresas, desde as caseiras até as grandes, com distribuição em todo o país. Estes produtos são passíveis de contaminação por diferentes micro-organismos que podem causar deterioração do produto ou acarretar o desenvolvimento de doenças, afetando a saúde humana. Dentre as principais análises microbiológicas que indicam contaminação em requeijão e doce de leite pastoso, destacam-se Coliformes a $30^{\circ} \mathrm{C}$ e Coliformes a $45^{\circ} \mathrm{C}$, Staphylococcus spp., além da detecção de fungos filamentosos e leveduras. Este trabalho teve como objetivo realizar mensalmente, análises microbiológicas do requeijão e do doce de leite pastoso produzidos por uma indústria de laticínios da cidade de Tocantins MG, no ano de 2007. De acordo com os resultados, pode-se observar que todas as amostras de requeijão e de doce de leite pastoso enquadraram-se aos padrões estabelecidos pela legislação vigente. Portanto, conclui-se que os dois produtos apresentaram boa qualidade microbiológica, estando, neste aspecto, adequados para a comercialização e consumo humano.

Palavras-chave: Avaliação microbiológica, Doce de leite e Requeijão.

\begin{abstract}
Curd is a typically Brazilian product, manufactured in the entire national territory, with some variations of technology and features. Another product of great importance is the dulce de leche, which production in Brazil is made by many companies, from home to large, distributed throughout the country. These products are susceptible to contamination by different microorganisms that can cause product deterioration or lead the development of diseases affecting human health. Among the main microbial contaminants in cheese and dulce de leche, we highlight the Fecal Coliforms $30^{\circ} \mathrm{C}$ and $45^{\circ} \mathrm{C}$, Staphylococcus spp. and filamentous fungi and yeasts. This study aimed to perform monthly, microbiological analysis of the cream cheese and dulce de leche produced by a dairy town of Tocantins - MG, in 2007. According to the results, one can observe that all samples of cheese and dulce de leche fitted to the standards established by the legislation.
\end{abstract}


Therefore, we conclude that the two products showed good microbiological quality, being in this respect, suitable for marketing and consumption.

Keyword: Microbiological evaluation, Curd and Sweet milk.

\section{INTRODUÇÃO}

A cadeia industrial do leite representa elevada expressão no setor alimentício. O leite, além de ser, um alimento indispensável na mesa da população (CAMPOS et al., 2003), é inegável a importante influência que a cadeia pecuária leiteira, exerce na economia do Brasil (COUTINHO, 2008). O leite e seus derivados são importantes fontes de nutrientes, sendo considerados os principais fornecedores de cálcio biodisponível necessário para a formação de massa óssea (CAMPOS et al., 2003). Esta riqueza nutricional faz com que o leite seja um meio propício ao crescimento de diversos grupos de micro-organismos.

Pode-se afirmar que a qualidade do produto final obtido depende da qualidade da matéria-prima. Deste modo, para a elaboração de queijos e outros derivados lácteos, como o doce de leite, deve-se utilizar leite de boa qualidade, tanto em relação ao aspecto microbiológico, quanto a sua composição físico-química, já que tais características influenciam consideravelmente no processo de fabricação (SANCHEZ, 2004).

No mundo existem diversos tipos de queijos, entre os quais estão os queijos fundidos. Essa tecnologia surgiu no início do século XX, a fim de inativar os processos microbianos e enzimáticos de queijos suíços e alemães, viabilizando a exportação para países de clima quente. Embora a aplicação tecnológica acarretasse vantagens, constatou-se o inconveniente da separação de gordura e proteína do produto. Para solucionar o problema originou-se um método que consistia em solubilizar o paracaseinato de cálcio da matéria-prima por calor, usando citrato de sódio como agente peptizante, obtendo assim o que foi chamado de queijo fundido (VAN DENDER, 1992).

Dentre os principais queijos fundidos está o requeijão, produto tipicamente brasileiro, originário de fabricações caseiras, como uma forma de aproveitamento do leite (ABIQ, 2004) sendo fabricado em todo o território nacional, com algumas variações de tecnologia e características pelas regiões de implantação (MUNCK e CAMPOS, 1984). De acordo com a legislação, entende-se por requeijão comum o produto obtido pela fusão da massa coalhada, cozida ou não, dessorada e lavada, proveniente da coagulação ácida e/ou enzimática do leite, opcionalmente adicionada de creme de leite e/ou manteiga e/ou gordura anidra de leite e/ou butteroil, podendo ser adicionado de condimentos, especiarias e/ou outras substâncias alimentícias (BRASIL, 1997a). Furtado e Neto (1994) complementam que este produto é fabricado a partir de leite desnatado, cru ou pasteurizado, com ou sem adição de cultura lática (fermentação lenta natural) e que a fusão é normalmente acompanhada pela a adição de sais fundentes. A utilização de sais fundentes é fundamental para evitar a separação de gordura e de água da mistura, o que iria acontecer naturalmente após o aquecimento, caso estes aditivos não fossem acrescentados (VAN DENDER, 2006).

Outros produtos de grande importância no setor lácteo são aqueles obtidos pelo processo de concentração do leite por evaporação, e segundo Hall e Hedrick (1971), a aplicação dos processos de concentração do leite através do uso de calor iniciou-se em 1856.

O doce de leite é um alimento proveniente do processo de concentração do leite, produzido e comercializado principalmente no Brasil e na Argentina (PERRONE, 2006). É um doce de cor castanho caramelado com diferentes consistências: cremosa ou pastosa, sem cristais sensorialmente perceptíveis; semi-sólida; sólida e parcialmente cristalizada. Como ingredientes opcionais, podem ser inseridos ao doce de leite, creme, sólidos de origem láctea, mono e dissacarídeos que substituam a sacarose em no máximo de $40 \% \mathrm{~m} / \mathrm{m}$, amidos ou amidos modificados em uma proporção não superior a $0,5 \mathrm{~g} / 100 \mathrm{~mL}$ no leite, cacau, chocolate, coco, amêndoas, amendoim, frutas secas, cereais e/ou outros produtos alimentícios isolados ou misturados em uma proporção entre $5 \%$ e $30 \% \mathrm{~m} / \mathrm{m}$ do produto final (BRASIL, 1997b).

Trata-se de um produto amplamente empregado como ingrediente para a elaboração de alimentos como confeitos, bolos, biscoitos, sorvetes e também consumido diretamente como sobremesa ou acompanhado de pão, torradas e queijo (DEMIATE et al., 2001). Assim como os demais produtos lácteos, o requeijão e o doce de leite são passíveis de contaminação por diferentes agentes etiológicos, que podem levar ao desenvolvimento de doenças. A presença de micro-organismos patogênicos e/ou suas toxinas nos alimentos, representa um risco potencial à saúde humana (PASSOS et al., 1996). A segurança alimentar é um desafio atual, devendo ser analisada ao longo de toda cadeia alimentar (BRITO et al., 2008). 
O exame microbiológico de um alimento pode ser realizado, entre outros motivos, para verificar sua qualidade microbiológica ou se este satisfaz determinados critérios microbiológicos. Os micro-organismos indicadores são pesquisados com o objetivo de verificar a qualidade higiêncio-sanitária dos alimentos (FRANCO e LANDGRAF, 1996). Os resultados das análises microbiológicas indicam a qualidade da matéria-prima empregada, a limpeza das condições de preparo do alimento e a eficiência do método de preservação. A escolha das análises microbiológicas e os métodos a serem adotados, para tais, são determinados de acordo com o tipo de alimento que está sendo analisado e pelo propósito específico da análise. Além disso, os tipos de micro-organismos que poderão estar presentes no alimento devem ser levados em consideração (HAJDENWURCEL, 1998).

Vários micro-organismos podem causar problemas quando presentes nos alimentos, tanto no ponto de vista econômico, quanto de saúde. Dentre esses micro-organismos estão os do grupo dos Coliformes (Totais e Termotolerantes), os Staphylococcus spp. e fungos filamentosos e leveduras. Os Coliformes têm como característica fermentar a lactose com posterior formação de gás. O principal micro-organismo que representante é a Escherichia coli, cujo habitat é o intestino de animais de sangue quente. Portanto, a detecção destas bactérias, pode indicar contaminação fecal (ADAMS e MOSS, 1997). A espécie Staphylococcus aureus é anaeróbia facultativa, forma cocos, ocorrendo isolados, aos pares e em aglomerados. Está frequentemente relacionada aos casos e aos surtos de intoxicação alimentar, devido à capacidade da maioria das suas cepas de produzir enterotoxinas. Em função do risco à saúde pública e a importância que a presença da enterotoxina representa em alimentos, estabeleceu-se em diversos países a obrigatoriedade de sua pesquisa e enumeração, como parte das ações de fiscalização sanitária de órgãos governamentais (GANDRA, 2003).

Quando o ambiente está insalubre, ele se torna um meio propício para o crescimento de muitos micro-organismos como fungos filamentosos e leveduras, que quando presentes nos alimentos podem favorecer a perda do produto, resultando em prejuízo para as indústrias, podendo causar alterações de sabor, cor, estufamento de embalagem nas prateleiras refrigeradas de comercialização. Embora não seja comum patogenia por parte destes micro-organismos em produtos lácteos, a presença deles, não deixa de ser um risco à saúde dos consumidores. Diante do exposto, este trabalho teve como objetivo avaliar qualidade microbiológica de requeijão e doce de leite pastoso produzido por uma indústria da cidade de Tocantins, MG.

\section{MATERIAL E MÉTODOS}

Para controle microbiológico do requeijão e doce de leite pastoso foram realizadas contagens de Coliformes a $35^{\circ} \mathrm{C}$, Coliformes a $45^{\circ} \mathrm{C}$ e contagem de Staphylococcus spp. além de fungos filamentosos e leveduras somente para doce de leite pastoso. As análises foram realizadas uma vez por mês em duplicatas, sendo feitas oito análises para coliformes a $35^{\circ} \mathrm{C}$, coliformes a $45^{\circ} \mathrm{C}$, bolores e leveduras, enquanto que, para os Staphylococcus coagulase positiva, realizaram-se onze análises no decorrer do ano de 2007. A metodologia de obtenção, preparo e análises microbiológicas das amostras foi executada segundo descrito por Silva et al. (1997).

\subsection{Obtenção e preparo das amostras de requeijão e doce de leite pastoso}

Para a realização deste trabalho foram coletadas amostras de requeijão e doce de leite pastoso de uma indústria de laticínios da cidade de Tocantins-MG, e transportadas para o Laboratório de Microbiologia de Alimentos do Instituto Federal de Educação, Ciência e Tecnologia do Sudeste de Minas Gerais - Campus Rio Pomba (IF Sudeste MG/RP). As amostras foram coletadas uma vez por mês em dias variados para não interferir no resultado, sendo este processo repetido durante todo o ano de 2007. Cada embalagem foi identificada e higienizada (água corrente, detergente e álcool etílico 70\%) para remoção dos contaminantes superficiais. Assepticamente, $25 \mathrm{~g}$ de cada amostra foram pesadas em balança GEHAKA ${ }^{\circledR}$ e transferidas para $225 \mathrm{~mL}$ da solução de citrato de sódio $2 \%$ (diluição $10^{-1}$ ) para homogeneização. A partir desta diluição foram realizadas outras diluições subseqüentes $\left(10^{-2}\right.$ e $\left.10^{-3}\right)$.

\subsection{Contagem de Coliformes a $30^{\circ} \mathrm{C}$ e Coliformes a $45^{\circ} \mathrm{C}$}

A contagem de coliformes totais (Coliformes a $30^{\circ} \mathrm{C}$ ) foi realizada pelo "método do número mais provável" (NMP). Alíquotas de $1 \mathrm{~mL}$ de cada diluição foram transferidas para tubos contendo $10 \mathrm{~mL}$ de 
caldo lauril sulfato triptose (LST) $\left(35,5 \mathrm{~g} \mathrm{~L}^{-1}\right)$ e incubadas em estufa durante $24 \mathrm{~h}$, a $35^{\circ} \mathrm{C}$, para observação do crescimento microbiano com produção de gás.

Em caso positivo, uma alçada carregada dos tubos LST foi transferida para o caldo verde brilhante bile (VB) $\left(40 \mathrm{~g} \mathrm{~L}^{-1}\right)$ para contagem dos coliformes totais. Os tubos de caldo VB foram incubados durante 24$48 \mathrm{~h}$ a $35^{\circ} \mathrm{C}$ para se observar o crescimento e a produção de gás.

O número de tubos VB positivos para crescimento e ocorrência de gás foi registrado para determinação do NMP/g através da tabela disponível em Silva et al. (1997). Outra alçada dos tubos LST positivos foi transferida para tubos contendo o caldo Escherichia coli (EC) $\left(37 \mathrm{~g} \mathrm{~L}^{-1}\right)$ para determinação dos coliformes fecais (Coliformes a $45^{\circ} \mathrm{C}$ ).

Os tubos com caldo EC foram incubados em banho-maria a $45,5^{\circ} \mathrm{C}$ durante $24 \mathrm{~h}$. Após este período analisou-se o crescimento e a produção de gás. O número de tubos positivos foi anotado e o NMP/g foi obtido utilizando-se os dados de Silva et al. (1997).

\subsection{Contagem de Staphylococcus coagulase positiva}

Para esta análise foram utilizas, as diluições $10^{-2}$ e $10^{-3}$, e inoculação de $0,1 \mathrm{~mL}$ em uma placa, para cada diluição, contendo Agar Baird-Parker (BP), previamente preparada e adicionado de emulsão de gema de ovo e telurito de potássio a $1 \%$. No entanto, na diluição $10^{-1}$, a inoculação foi realizada em três placas com o referido meio, sendo que duas foram inoculadas com $0,3 \mathrm{~mL}$ e uma com $0,4 \mathrm{~mL}$.

Os inóculos foram espalhados com o auxílio de uma alça de Drigalski, das placas de maior para as de menor diluição, até que houvesse a completa absorção do excesso de líquido. Após secas, as placas foram incubadas, invertidas, a $35^{\circ} \mathrm{C}$ por 48 horas, em estufa de incubação (BOD Quimis ${ }^{\circledR}$ Q-315d) Para a confirmação, foi adotado o teste de catalase.

Foram selecionadas cinco colônias típicas para a inoculação e emulsão em tubos correspondentes contendo 5,0 mL de Caldo Infusão Cérebro Coração (BHI, Brain Heart Infusion Agar). Em paralelo, uma alçada foi transferida desta emulsão para tubos contendo $10,0 \mathrm{~mL}$ de Agar Tripticase de Soja (TSA, Trypticase Soy Agar) inclinado.

Os tubos contendo os dois meios foram incubados a $35^{\circ} \mathrm{C}$ por 24 horas. Para o teste de catalase, adicionou-se 1,0 $\mathrm{mL}$ na rampa dos tubos de TSA. Nos tubos que apresentaram borbulhamento imediato, a presença de Staphylococcus spp. foi considerada positiva.

Para o teste de coagulase, $0,2 \mathrm{~mL}$ de cada tudo de BHI foi transferido para outro contendo $0,5 \mathrm{~mL}$ de plasma de coelho com ácido etilenodiaminotetracético (Plasma-EDTA), posteriormente misturados com movimentos de rotação. Os tubos foram incubados em banho-maria a $37^{\circ} \mathrm{C}\left(\mathrm{Tecnal}^{\circledR}\right)$ e, a cada hora, durante quatro horas, foi observado se haveria a formação de coágulos.

\subsection{Contagem de Fungos filamentosos e Leveduras}

Para a realização desta análise foi adotado o "método de plaqueamento em superfície". Placas de Petri contendo 20-25 mL de Agar Batata Dextrose (PDA, Potato Dextrose Agar) (39g L $\mathrm{g}^{-1}$ ), autoclavado durante 15 minutos a $121^{\circ} \mathrm{C}$ e acidificado com $1 \mathrm{~mL}$ de ácido tartárico $10 \%$ para cada $100 \mathrm{~mL}$ de PDA, foram inoculadas com alíquotas de $0,1 \mathrm{~mL}$ de cada diluição. As placas não invertidas foram incubadas em estufa (BOD Quimis ${ }^{\circledR}$ Q-315d) durante 5 dias a $25^{\circ} \mathrm{C}$.

\section{RESULTADOS E DISCUSSÃO}

Todas as amostras apresentaram NMP de coliformes totais e termotolerantes dentro dos padrões estabelecidos na legislação pertinente (Tabelas 1 e 2). Resultados semelhantes foram encontrados por Lourenço et al. (2002) ao realizarem análises microbiológicas em três tipos de requeijão de leite de búfala, cujos resultados variaram de $<3$ a $240 \mathrm{NMP} / \mathrm{g}$. De acordo com os resultados das análises microbiológicas das amostras de requeijão (Tabela 1), todas atenderam aos padrões estabelecidos pela Portaria $\mathrm{N}^{\mathbf{0}} 359$ (BRASIL, 1997a), tanto para as análises de coliformes, quanto para Staphylococcus spp.. Lourenço et al. (2002) analisaram quatro tipos de queijos, sendo dois de búfalas e dois de vacas, dos quais um de cada era artesanal e outro fabricado com a utilização de tecnologia. 
Tabela 1 - Padrão microbiológico do requeijão produzido por uma indústria da cidade de Tocantins, MG.

Coliformes $35^{\circ} \mathrm{C}$

$(\mathrm{NMP} / \mathrm{g})$
Coliforme $45^{\circ} \mathrm{C}$

$(\mathrm{NMP} / \mathrm{g})$
Staphylococcus coagulase positiva

(UFC/g)

\begin{tabular}{lll}
\hline$<0,3$ & $<0,3$ & $<1 \times 10^{1}$ \\
$<0,3$ & $<0,3$ & $<1 \times 10^{1}$ \\
$<0,3$ & $<0,3$ & $<1 \times 10^{1}$ \\
$<0,3$ & $<0,3$ & $<1 \times 10^{1}$ \\
$<0,3$ & $<0,3$ & $<1 \times 10^{1}$ \\
$<0,3$ & $<0,3$ & $<1 \times 10^{1}$ \\
$<0,3$ & $<0,3$ & $<1 \times 10^{1}$ \\
$<0,3$ & $<0,3$ & $<1 \times 10^{1}$ \\
$<0,3$ & $<0,3$ & $<1 \times 10^{1}$ \\
$<0,3$ & $<0,3$ & $<1 \times 10^{1}$ \\
$<0,3$ & $<0,3$ & $<1 \times 10^{1}$ \\
\hline
\end{tabular}

Conforme demonstrado na Tabela 1, a contagem de Staphylococcus coagulase positivo apresentou valores abaixo de $1,0 \times 10^{1}$, visto que, de acordo com Sena (2000), estes micro-organismos são capazes de produzir toxinas independentemente de serem coagulase positiva ou não. A presença de enterotoxinas estafilocócicas no leite e nos produtos lácteos tem sido constatada em diversos estudos. Rosec et al. (1997) em estudos com queijos elaborados a partir de leite cru, observaram a presença de toxina do tipo $\mathrm{C}$ em $73,7 \%$ das 61 amostras analisadas.

Masud et al. (1993) em estudos com 48 linhagens enterotoxigênicas de S. aureus, isoladas de produtos lácteos, observaram que pelo menos 16 delas produziram mais de um tipo de enterotoxina. A enterotoxina mais comumente encontrada foi a do tipo A, seguida dos tipos B, D e C.

Neste estudo, não foi realizada contagem de fungos filamentosos e leveduras nas amostras de requeijão, uma vez que, a legislação em vigor não estipula valores para a contagem desses micro-organismos em requeijão.

Tabela 2 - Padrão microbiológico do doce de leite pastoso produzido por uma indústria da cidade de

\begin{tabular}{cccc}
\multicolumn{4}{c}{ Tocantins, MG. } \\
\hline $\begin{array}{c}\text { Coliformes } 35^{\circ} \mathrm{C} \\
\mathrm{NMP} / \mathrm{g}\end{array}$ & $\begin{array}{c}\text { Coliformes } 45^{\circ} \mathrm{C} \\
\text { NMP/g }\end{array}$ & $\begin{array}{c}\text { Staphylococcus coagulase } \\
\text { positiva (UFC/g) }\end{array}$ & $\begin{array}{c}\text { Bolores e Leveduras } \\
(\mathrm{UFC} / \mathrm{g})\end{array}$ \\
\hline$<0,3$ & $<0,3$ & $<1 \times 10^{1}$ & $<1 \times 10^{1}$ \\
$<0,3$ & $<0,3$ & $<1 \times 10^{1}$ & $<1 \times 10^{1}$ \\
$<0,3$ & $<0,3$ & $<1 \times 10^{1}$ & $<1 \times 10^{1}$ \\
$<0,3$ & $<0,3$ & $<1 \times 10^{1}$ & $<1 \times 10^{1}$ \\
$<0,3$ & $<0,3$ & $<1 \times 10^{1}$ & $<1 \times 10^{1}$ \\
$<0,3$ & $<0,3$ & $<1 \times 10^{1}$ & $<1 \times 10^{1}$ \\
$<0,3$ & $<0,3$ & $<1 \times 10^{1}$ & $<1 \times 10^{2}$ \\
$<0,3$ & $<0,3$ & $<1 \times 10^{1}$ & $<1 \times 10^{2}$ \\
$<0,3$ & $<0,3$ & $<1 \times 10^{1}$ & $<1 \times 10^{2}$ \\
$<0,3$ & $<0,3$ & $<1 \times 10^{2}$ \\
$<0,3$ & $<0,3$ & $<1 \times 10^{1}$ & $<1 \times 10^{2}$ \\
\hline
\end{tabular}

Os resultados obtidos para a contagem de coliformes totais e termotolerantes (NMP) e Staphylococcus coagulase positiva (Tabela 2), indicam que todas as amostras de doce de leite pastoso atenderam aos padrões preconizados pela Portaria n. 354 de 04 de setembro de 1997 (BRASIL, 1997b). 
BRITO et al. (2008) avaliaram em seu trabalho as condições higiênico-sanitárias de amostras de doce de leite fabricado por uma indústria do Município de João Pessoa - PB e obtiveram resultados semelhantes para coliformes termotolerantes e para os Staphylococcus coagulase positiva. Portanto, o doce de leite pastoso foi processado em condições higiênico-sanitárias, o que justifica a baixa contagem de microorganismos do grupo coliformes e $S$. coagulase positivo.

Em relação à contagem de fungos filamentosos e leveduras, valores entre $<1,0 \times 10^{1} \mathrm{e}<1,0 \times 10^{2}$ foram constatados (Tabela 2). Portanto, atenderam os padrões estabelecidos pela legislação vigente (BRASIL, 1997b). Beloti et al. (2001) citaram que os fungos filamentosos e leveduras são muito importantes como indicadores de contaminação na cadeia produtiva do leite, podendo ser considerados como um fator de contaminação quando isolados de água residual nos utensílios e equipamentos. A presença desses micro-organismos no produto pode levar à redução no tempo de vida de prateleira, modificação de suas características sensoriais e até mesmo deterioração.

\section{CONCLUSÃO}

De acordo com os resultados obtidos neste estudo, conclui-se que todas as amostras de requeijão e de doce de leite pastoso apresentaram boa qualidade microbiológica em relação às condições analisadas.

\section{REFERÊNCIAS BIBLIOGRÁFICAS}

ADAMS, M.R.; MOSS, M.O. Microbiologia de Los alimentos. Zaragoza: Acriba, 1997.

BELOTI, V.; BARROS, M.A.F.; SANTANA, E.H.W.; PEREIRA, M.S.; MORAES, L.B.; GUSMÃO, V.V. Utilização de bolores e leveduras como indicadores de contaminação na cadeia produtiva de leite em propriedades da região de Londrina, Paraná. XXI Congresso Brasileiro de Microbiologia. Foz do Iguaçu-PR, p.377, 2001.

BRASIL(a), Ministério da Agricultura e do Abastecimento. Secretaria de Defesa Agropecuária. Departamento de Inspeção de Produtos de Origem Animal. Regulamentos Técnicos de Identidade e Qualidade de Leite e Produtos Lácteos. Portaria $\mathrm{n}^{\circ} 359$. Regulamento técnico para fixação de identidade e qualidade do requeijão cremoso ou requesón. Diário Oficial da República Federativa do Brasil. Brasília DF, 1997.

BRASIL(b), Ministério da Agricultura, Pecuária e Abastecimento. Departamento de Inspeção de Produtos de origem Animal. Portaria n. 354, de 04 de setembro de 1997. Regulamento Técnico para Fixação de Identidade e Qualidade de Doce de Leite. Diário Oficial da República Federativa do Brasil, Brasília DF, 1997.

BRITO, G.G.A.P.; FERREIRA D.N.; RAMOS, N.P; SILVA, P.S.; SILVEIRA, E.O.; CABRAL, T.M.A.; NASCIMENTO, G.J. Aspecto sanitário de 10 amostras de doce de leite fabricado por uma indústria do município de João Pessoa - PB. Anais do XI Encontro de iniciação à docência. In: Encontro de iniciação à docência. João Pessoa PB, 2008.

CAMPOS, L.M.A; LIPHAUS, B.L; SILVA, C.A.A; PEREIRA, R.M.R. Osteoporose na infância e na adolescência. Jornal de Pediatria, Rio de Janeiro, v.79, n.6, p.481-488, 2003.

COUTINHO, P. Planejamento e gestão estratégica do sistema agroindustrial do leite no estado de São Paulo. InterJornal-Brasil. Agência Sebrae de Notícias, 2008.

DEMIATE, I.M; KONKEL, F.E; PEDROSO, R.A. Avaliação da Qualidade de Amostras Comerciais de Doce de Leite Pastoso - Composição Química. Revista Ciência e Tecnologia de Alimentos. vol.21 n.1 Campinas Jan./Apr. 2001.

FURTADO, M.M.; LOURENÇO NETO, J.P.M. Tecnologia de queijos. São Paulo: Dipemar, 1994. 118p. 
FRANCO, B.D.G.M.; LANDGRAF, M. Microbiologia dos alimentos. São Paulo: Atheneu, 1996, 182p.

HAJDENWURCEL, J.R. Atlas de Microbiologia de Alimentos. Volume 1, São Paulo, Fonte Comunicação, 1998.

HALL, C.W.; HEDRICK, T.I. Drying of Milk and Milk Products. 2nd edn. AVI Publ. Co., Westport, CT, U.S.A, 1971.

LOURENÇO, L.F.H.; Sousa, C.L.; Simão Neto, M.; Lourenço Júnior, J.B. Análise microbiológica do requeijão marajoara no norte do Brasil. Revista Higiene Alimentar, 16(96):55-59, 2002.

MASUD, T.; ALI, A.M.; SHAH, M.A. Enterotoxigenicity of Staphylococcus aureus isolated from dairy products. Aust J Dairy Technol, n.48, p.30-32, 1993.

MUNCK, A.V.; CAMPOS, W.A. Requeijão: um produto brasileiro. Informe Agropecuário, v.10, p.35-38, 1984.

PASSOS, M.H.C.R.; KUAYE, A.Y. Relato de surtos de intoxicação alimentar provocada por consumo de bolo contaminado por Staphylococcus aureus: importância da higiene dos manipuladores e condições de conservação do alimento na prevenção da doença. Revista do Instituto Adolfo Lutz, v. 56, n. 1, p. 71-76, 1996.

PERRONE, I.T. Efeito da nucleação secundária sobre a cristalização do doce de leite. Dissertação de Mestrado (Ciência dos Alimentos). Lavras: UFLA, 2006.49 p.

ROSEC, J.P. et al. Enterotoxin production by staphylococci isolated from foods in France. Int J Food Microbiol, v.35, p.213-221, 1997.

SANCHEZ, M.A.P. Influência da matéria prima no fabrico de queijo de cabra. Relatório do Trabalho de Fim de Curso de Engenharia Alimentar. Lisboa, 2004.

SENA, M. J. Perfil epidemiológico, resistência a antibióticos e aos conservantes nisina e sistema lactoperoxidase de Staphylococcus spp. isolados de queijos coalho comercializados em Recife-PE. 2000. 75 f. Tese (Doutorado em Ciência Animal) - Universidade Federal de Minas Gerais, Belo Horizonte.

VAN DENDER, A.G.F. Princípios básicos da fabricação de queijos fundidos de requeijão cremoso. Leite $e$ Derivados, v. 1, n. 3, p. 26, 1992.

GANDRA, E.A. Identificação de Staphylococcus aureus, S. intermedius e S. hyicus através de testes bioquímicos e da amplificação por PCR de seqüências dos genes coa E nuc. Dissertação de Mestrado (Ciência e Tecnologia Agroindustrial). UFP, Pelotas-RS, 2003.

SILVA, N.; JUNQUEIRA, V.C.A.; SILVEIRA, N.F.A. Manual de métodos de análise microbiológica de alimentos, 2a. edição. São Paulo: Ed. Varela, 1997. 